\title{
Prácticas Sociocomunitarias de estudiantes de Geografía con organizaciones sociales de Sierras Chicas, Córdoba
}

\author{
Beatriz Ensabella \\ bettyensabella@gmail.com
}

Sergio Chiavassa

sachapig@yahoo.com.ar
Departamento de Geografía, Facultad de Filosofía y Humanidades, Universidad Nacional de Córdoba, Argentina.
A 100 años de la Reforma Universitaria de 1918 /

Intervenciones

RECEPCIÓN: 29/06/17

ACEPTACIÓN FINAL: 02/10/17

\section{Resumen}

El artículo hace eje en una experiencia pedagógica, la Práctica Sociocomunitaria (PSC), llevada a cabo por profesores, alumnos y ayudantes del Seminario Organización territorial II (Rural), materia de la carrera de Licenciatura en Geografía perteneciente a la Facultad de Filosofía y Humanidades de la Universidad Nacional de Córdoba.

La PSC es una experiencia pedagógica innovadora, que acerca a los alumnos de carreras de grado universitario al campo social de los conflictos territoriales. Se trabaja conjuntamente entre la cátedra y sus estudiantes con organizaciones sociales, en este caso de tipo ambientalistas, movilizadas en defensa del suelo, el bosque y el agua, en localidades de las Sierras Chicas de Córdoba (Argentina). Creemos que la PSC permite la co-construcción del conocimiento y acorta distancias entre la Universidad y la sociedad. Dadas estas valiosas características, presentamos en el artículo nuestra experiencia realizada con colectivos de Sierras Chicas durante los años 2015, 2016, y $1^{\circ}$ cuatrimestre de 2017, acompañada de una serie de reflexiones sobre el trabajo de campo geográfico, la relación Universidad-comunidad, las maneras diversas de aprender fuera del contexto áulico, entre otras cuestiones.

\section{Palabras clave}

- Organizaciones sociales

- Conflictos ambientales

- Territorio

- Práctica Sociocomunitaria

\section{Resumo}

O eixo de artigo basa se em uma experiência pedagógica, a practice sócio-comunitária (PSC), coordinada por professores, alunos e assistentes da matéria Rural, o bacharel em Geografia, à Faculdade de Filosofia e Humanidades da Universidade Nacional de Córdoba, em cidades do Sierras Chicas de Córdoba (Argentina).

A PSC é uma experiência que permite abordagen o campo social dos conflitos territoriais, com organizações de base sociais motorizados, que conhecem os seus problemas locais em profundidade. A PSC pretende ser uma extensão da realidade do conhecimento em que vários agentes sociais, viven e lutan. Acreditamos que o PSC permite que o co-construção de conhecimentos e encurta distâncias entre a universidade ea sociedade. Dadas estas valiosas características, presentamos no artigo, a nossa experiência realizada com grupos de Sierras Chicas durante os anos de 2015, 2016 e $1^{\circ}$ semestre de 2017, acompanhado por uma série de reflexões sobre o trabalho de campo geográfico, as várias maneiras de aprender fora do contexto da aula, entre outras questões.

Palavras-chave

- Conflitos sociais

- Conflitos ambientais

- Território

- Practice sócio-comunitária
Para citación de este artículo

Ensabella, B. y Chiavassa, S. (2017). Prácticas

Sociocomunitarias de estudiantes de Geografía con

organizaciones sociales de Sierras Chicas, Córdoba. Revista

+E versión en línea, 7(7), 308-319. Santa Fe, Argentina:

Ediciones UNL. 


\section{Introducción}

Deberíamos comenzar por presentar los objetivos que impulsan las acciones extensionistas que vamos a compartir en este artículo. Sin embargo, nuestras inquietudes, es bueno decirlo desde un principio, persiguen propósitos que van más allá de la extensión en el ámbito universitario y tienen que ver con nuestra opción de vida, nuestra manera de estar en el mundo.

Por eso, y atendiendo sobre todo a esta última cuestión, es que desde siempre nos preocuparon las cuestiones ambientales en nuestros entornos de vida y tratamos de involucrarnos en tareas de extensión desde la cátedra y desde el equipo de investigación en el que estamos insertos como forma de contribuir en su defensa. ¿De qué nos serviría ser militantes, proteger el bosque, el suelo, el agua, ser de alguna manera "ambientalistas", si en la docencia universitaria mantenemos el formato tradicional de clases en el aula y, como máximo, planificamos una salida de campo? Más aún, nos preguntamos: ¿se conoce sobre el ambiente, sobre los recursos naturales, sobre nuestros derechos como ciudadanos mediante el abordaje de aspectos y perspectivas teóricas casi como única posibilidad de aprender y de formar profesionales? Creemos que no, que es necesario salir al campo, entrar en contacto con la gente y junto con la gente aprender y, si es necesario, organizarse y luchar codo a codo. También siempre nos planteamos que la docencia y la investigación son incompletas si no se hace extensión, sobre todo considerando que nuestras actividades se llevan adelante en una carrera de Geografía, donde el trabajo de campo es fundamental a la hora de analizar cualquier problemática de la realidad.

Entre tanto, y mientras nos cuestionábamos y desarrollábamos actividades de docencia e investigación, el modelo de universidad para nuestros países, también fue de a poco virando desde uno de tipo academicista hacia uno más involucrado y en contacto con la sociedad. En ese marco de renovación, se reglamentó la Práctica Sociocomunitaria (PSC) para las carreras de grado en la Facultad de Filosofía y Humanidades (FFyH) de la Universidad Nacional de Córdoba (UNC).

Sobre la base de las preocupaciones, pensamientos y condiciones contextuales enunciadas, profesores, estudiantes y ayudantes nos hemos convocado en grupo para sostener desde el año 2015 una PSC en la cátedra de Seminario de Organización Territorial II (Rural) de la carrera de Geografía de la mencionada Facultad. En este artículo queremos compartir nuestras experiencias a los fines de impulsar desde estas acciones todas aquellas actividades que fortalezcan el extensionismo en nuestras universidades.

\section{Enmarcando la extensión para el grado en las PSC}

Hablábamos de articular docencia, investigación y extensión, desde experiencias que incluyan a los estudiantes de carreras de grado. Efectivamente, eso es lo que se logró desde la Secretaría de Extensión de la FFyH en el año 2013 a través de la reglamentación de lo que se denomina PSC, entendida como el trabajo coordinado desde una cátedra con colectivos y organizaciones sociales externos al ámbito universitario que trabajan en un territorio concreto.

Consisten en propuestas curriculares que apuestan a experiencias educativas en estrecho vínculo con la comunidad orientadas a coordinar acciones en los procesos de reflexión crítica, identificación de problemas, diagnósticos o propuestas de resolución.

Pero: ¿cómo se llegó a jerarquizar las prácticas extensionistas en las carreras de grado? Creemos que una serie de condiciones contextuales las propiciaron, además de la voluntad política expresada por las autoridades de la Facultad en ese momento. Respecto del contexto, recordemos que la extensión ocupó un lugar marginal en el ámbito académico y en cierto modo esta situación continúa a pesar de los importantes avances realizados. Sin embargo, el surgimiento de nuevos paradigmas sobre cómo entender la construcción del conocimiento, el advenimiento de gobiernos progresistas y populares en los países de América Latina, la necesidad de contar con el apoyo local en la puesta en marcha de emprendimientos productivos, el reconocimiento de validar una amplia gama de derechos ciudadanos y la necesidad de disminuir las afectaciones ambientales de amplias zonas del planeta, son algunos de los factores que incidieron para que comenzara a gestarse un modelo de universidad más vinculado con los problemas concretos de las comunidades. Ya lo dice Boaventura de Sousa Santos en 2005 al hablar de la necesidad de reformar la universidad buscando

"alternativas de investigación, de formación, de extensión y de organización que apunten hacia la democratización del bien público universitario, es decir, para la contribución específica de la universidad en la definición y solución colectiva de los problemas sociales, nacionales y globales". $(2005: 25)^{1}$

La UNC no estuvo ajena a este proceso de cambio de las funciones universitarias tal como proponía Santos y, a partir de los primeros años del 2000 se fueron revalorizando las tareas de extensión en casi todas las unidades académicas, entendiendo la integralidad de las funciones universitarias. Políticas enfocadas al impulso hacia diversos proyectos y programas de extensión, con mayor
1) Boaventura de Sousa Santos, cuando en 2005 escribe:"La universidad en el siglo XXI. Para una reforma democrática y emancipadora", lo que en realidad hace en la primera parte, es una denuncia al sistema público universitario, en el sentido de que la universidad había servido cada vez más, a los intereses del capitalismo (fruto de una modernidad eurocéntrica, racionalista, economicista, tecnocrática y generadora de desequilibrios y desigualdades) y de este modo, había contribuido a veces sin desearlo, con la exclusión social, la inequidad, volviéndose antidemocrática. Cuando Santos imagina cómo debería ser la universidad del futuro, propone entre otras alternativas de solución necesarias, otorgarle un lugar central a las tareas de extensión como una manera de achicar la brecha entre la ciencia, el conocimiento académico y los problemas y necesidades reales de la sociedad, y así contribuir a la ampliación de una vida democrática. 


\section{6}

\section{la pregunta claves es, ¿se pueden establecer diálogos entre formas de conocimiento que en principio responden a lógicas y necesidades que no necesariamente coinciden?}

carga presupuestaria y mayor cantidad de agentes universitarios involucrados, son la prueba de ello. ${ }^{2}$

En ese marco, la FFyH comenzó a diseñar un proyecto concreto de intervención en el territorio, pensado desde las cátedras como propuesta curricular de grado, en las diversas carreras que están dentro de su órbita. Fue así que, en el año 2013, se aprobó el

Reglamento de PSC que:

"emerge de la necesidad de institucionalizar nuevos modos de formación para los estudiantes; y como modalidad se enmarca en políticas que la UNC y la FFYH vienen desarrollando en torno a la jerarquización de la extensión y a la integralidad de las funciones universitarias (...) la PSC se inserta en el espíritu de la Universidad en cuanto trata de promover la actuación del universitario en el seno del pueblo al que pertenece, destacando su sensibilidad para los problemas de su época y las soluciones de los mismos [a la vez que trata de] proyectar su atención permanente sobre los grandes problemas y necesidades de la vida nacional, colaborando desinteresadamente en su esclarecimiento y solución". (Res HCD № 226/2013)

El proyecto de PSC, tal como se pensó para la FFyH y como versa en el reglamento, prevé dos modalidades: como espacios curriculares específicos o como parte de los ya existentes, de manera de permitir que esta experiencia sirva como antecedente para futuras modificaciones de los planes de estudios de las carreras de la Facultad.

Conceptualmente, son PSC aquellas instancias de formación llevadas a cabo en territorio, realizadas en coordinación con colectivos y organismos sociales comunitarios, públicos y privados con fines sociales, externos al ámbito universitario, que se orientan a coordinar acciones en los procesos de reflexión crítica, identificación de problemas, diagnósticos o propuestas de resolución de problemáticas relativas a las áreas de competencia de la FFyH. Consisten en propuestas curriculares que apuestan a experiencias educativas con un estrecho vínculo con la comunidad, conjugándose en procesos de enseñanza de práctica situada, que articulen las discusiones propias del campo teórico, con las problemáticas sociales y políticas del contexto de intervención. En síntesis, a través de la PSC se pretende aportar a la reflexión e intervención, sobre los problemas sociales de nuestra comunidad, de manera conjunta con diferentes organizaciones sociales. Sobre las dimensiones y las modalidades específicas que adquirió la PSC que exponemos en este artículo, volveremos más adelante, cuando avancemos sobre la experiencia concreta. Ahora nos interesa el sustento pedagógico de la PSC referido a la construcción del conocimiento, a los modos diversos de aprender y de saber y a la posibilidad de un aprendizaje de labor colaborativa entre los estudiantes y las organizaciones locales.

\section{La PSC como posibilidad de aprendizaje colectivo}

Es posible anclar la experiencia de la PSC en supuestos básicos sobre el conocimiento, los saberes, los aprendizajes, lo que se promueve desde la apertura de un pensamiento epistémico que decide "colocarse ante las circunstancias de la realidad" (Zemelman, 2011).

Se trata de una forma de construir conocimiento con los sujetos, colectivos o comunidades, en términos de una ecología de saberes
SUMA 400 de 2013, que tuvo como objetivo: aportar al fortalecimiento de las capacidades de gestión locales colaborando con los municipios, comunas y organizaciones populares de la provincia de Córdoba en el diseño de políticas públicas centradas en la construcción de una ciudadanía social. 
el aprendizaje colaborativo es un proceso complejo, principalmente para los que llegamos desde una formación académica, por eso requiere transitar un camino de desaprendizaje-reaprendizaje
(De Sousa Santos) recuperando in situ las prácticas y los saberes locales. Se busca revalorizar y poner en diálogo saberes que se "piensan" diferentes, aquellos propios de los académicos y los que construyen las comunidades.

Es evidente que en el encuentro con los otros se entrecruzan las expectativas y saberes de los universitarios, con las expectativas y saberes de la comunidad. Ahora bien, la pregunta claves es, ¿se pueden establecer diálogos entre estas formas de conocimiento, que planteados en términos situacionales, en principio responden a lógicas y necesidades que no necesariamente coinciden? Siendo esto así, ¿cuáles son los distintos modos en que se pueden asumir estos diálogos? Cuando decimos "pensamos", ¿ese "pensamos" no se reduce en muchas ocasiones a una racionalización que desde las prácticas y conocimientos que se construyen en la universidad, excluyen otras dimensiones de la experiencia (qué le pasa a la gente de la comunidad, cómo lo vive, qué siente, etc.). Entonces, ¿qué más deberíamos incorporar a este "pensamos" para que pueda dar cuenta de estos conocimientos territorializados? En definitiva, estas últimas preguntas dirigen a ese punto siempre desigual, asimétrico, en el que parecen situarse los principios de autoridad, legitimidad y cientificidad de las prácticas de producción de conocimiento alumbrando algo bastante simple pero que todavía nos debe llenar de asombro: ¿por qué nos cuenta tanto admitir como conocimiento esos sentidos comunes de la gente del lugar? Las formas en que usan, trabajan, se movilizan, hablan, nos están dando cuenta de un saber sobre el territorio que muchas veces no es tomado como conocimiento válido por la academia, pero en el caso de la PSC es la clave que permite esa co-construcción.
En tanto asume esas asimetrías, el aprendizaje colaborativo es un proceso complejo, principalmente para los que llegamos desde una formación académica, por eso requiere transitar un camino de desaprendizaje-reaprendizaje, deconstrucción y reconstrucción de nuevo conocimiento y nuevas metodologías. Y esto debemos trabajar con los alumnos en las instancias previas y posteriores al contacto con las organizaciones sociales, en una actitud permanente de reflexividad.

La PSC implica una metodología de aprendizaje rigurosa pero que se construye/piensa y ejecuta en conjunto con "el otro", en la que los diversos actores involucrados participan a lo largo de todo el proceso. La situación metodológica ideal es que se planteen agendas de investigación, se rastreen datos y se elabore información, se redacten resultados, se difundan avances, donde participen todos los miembros de la comunidad y de la academia conjuntamente. "Aceptar el diálogo de saberes al que aludíamos recién, implica reconocer que en una PSC, estamos discutiendo y construyendo sentidos" (Chiavassa, 2009:94. Resaltado en el original).

A través del intercambio producido con la PSC se conoce el territorio, se experimenta el trabajo de campo, se aprende a construir conocimiento desde la fusión entre lo experiencial y lo académico-formal. A su vez, se enriquecen los marcos teóricos de los sujetos involucrados, tanto los locales como los universitarios al intercambiar conceptos ordenadores sobre los problemas y conflictos de la realidad.

Para acercarnos esta co-construcción de conocimiento es necesario que asumamos que "los modos de conocer de los colectivos sociales son tan legítimos como aquellos conocimientos
3) Una de esas opciones es crear espacios nuevos como seminarios, talleres, etc., en los que las PSC organiza la totalidad de la propuesta y además, puede ser propia de cada carrera o transversal amás deuna carrera, área o ciclo. La otra manera que es la que nosotros asumimos y presentamos en el artículo, es insertar la Práctica como propuesta curricular (obligatoria u optativa) de las carreras que se dictan en la FFyH. Para mayores especificaciones consultar Reglamento disponible en: http://www. ffyh.unc.edu.ar/extension/wp-content/ uploads/2013/10/RES_226_2013.pdf 
y saberes que están objetivados y que, por tal, están organizados formalmente en sistemas de conceptos y teorías" (Zaffaroni y Juárez, 2014:112).

La manera de conocer de los colectivos sociales tiene su correlato a nivel de la práctica social en vinculación con "la capacidad de acción histórica, esto es, de la posibilidad de los actores individuales y colectivos de crear y modificar normas, valores, significados, en el contexto de una determinada sociedad" (Vasilachis, 1999:7).

Estamos sosteniendo que la co-construcción del conocimiento implica, acepta, sostiene que cada persona entiende y es capaz de explicar o argumentar desde sus propios marcos referenciales sobre la realidad social y de su comunidad. Dicho en otras palabras, "el paradigma interpretativo otorga valor de verdad a las diferentes formas en que los hablantes representan discursivamente la realidad" (Vasilachis, 1999:9).

Desde esta perspectiva, uno de los ejes en los cuales se apoya nuestra PSC es la necesidad de producir conocimiento situado, capaz de recuperar la importancia del lugar, del territorio como una categoría del "estar y del pensar" (Escobar, 2008). Lo que procuramos es acercar a los estudiantes a las múltiples lógicas locales de producción de culturas e identidades, prácticas ecológicas y económicas, que emergen de diversas y distantes comunidades, en este caso desde las organizaciones sociales de Sierras Chicas de Córdoba con las que realizamos nuestras Prácticas.

\section{Ubicación del área de la PSC}

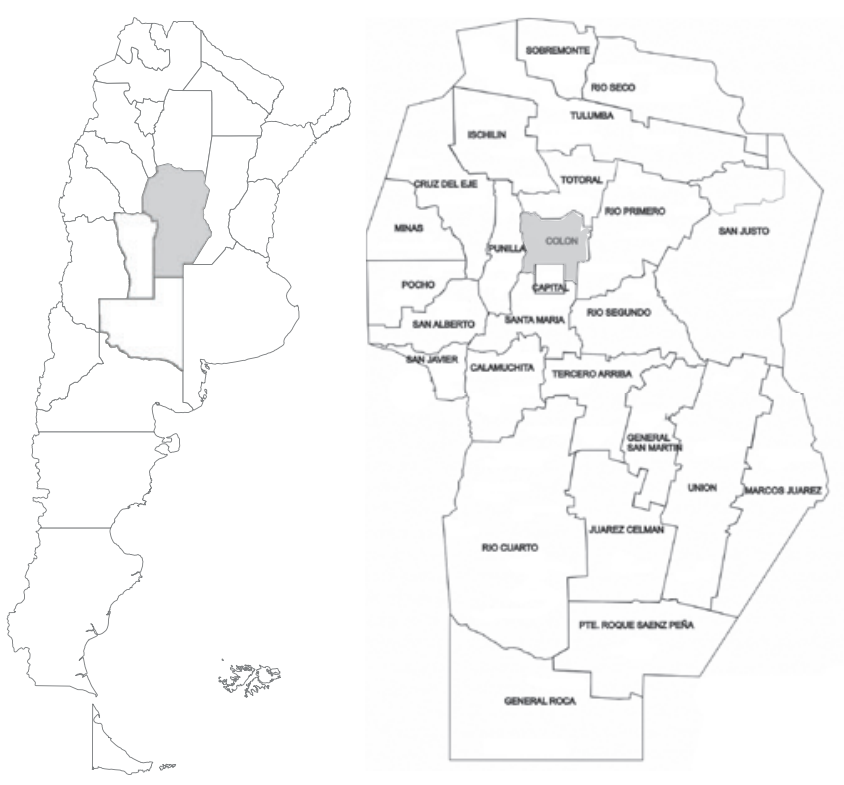

Las localidades donde se realiza la Práctica están ubicadas en el departamento Colón de la provincia de Córdoba, limítrofe al norte de la ciudad capital.

\section{Acercándonos al territorio de las PSC}

En primer término, queremos presentar la modalidad que adquirió nuestra PSC desde lo académico.

La Facultad ofrece diversas maneras de insertar la PSC en el grado, ${ }^{3}$ y nosotros optamos por incorporarla a la propuesta curricular de Geografía Rural, materia obligatoria y específica, de $3^{\circ}$ año de la carrera de Licenciatura en Geografía. Como la propuesta pedagógica debe incorporar un determinado número de horas de trabajo de los alumnos en terreno, la integramos a las clases prácticas de la materia. Estos espacios desarrollan en profundidad, y en horario independiente al de las clases teóricas, algunos de los temas del programa. Con relación a las temáticas, fue posible anclar la PSC en la unidad 4 del programa: "Problemáticas, tensiones y conflictos en los espacios periurbanos y rurales".

En efecto, el territorio de las Prácticas es una región periurbana y rural, caracterizada por problemas ambientales, principalmente relacionados con el uso del suelo, el desmonte y los recursos hídricos. Se ubica en localidades de las Sierras Chicas de Córdoba, pertenecientes al Área Metropolitana, a distancias de hasta $37 \mathrm{~km}$ de la capital, lo que posibilita los desplazamientos. Concretamente, las Prácticas hasta el momento se articularon con dos organizaciones sociales de Sierras Chicas, la Mesa del Agua y Ambiente (MAyA) de La Granja, experiencias realizadas en 2015 y 2016 y la última desarrollada en el $1^{\circ}$ cuatrimestre de 2017, con la Asamblea por el Bosque y la Vida de Villa Allende. En los siguientes mapas se puede ver la ubicación de los colectivos sociales donde

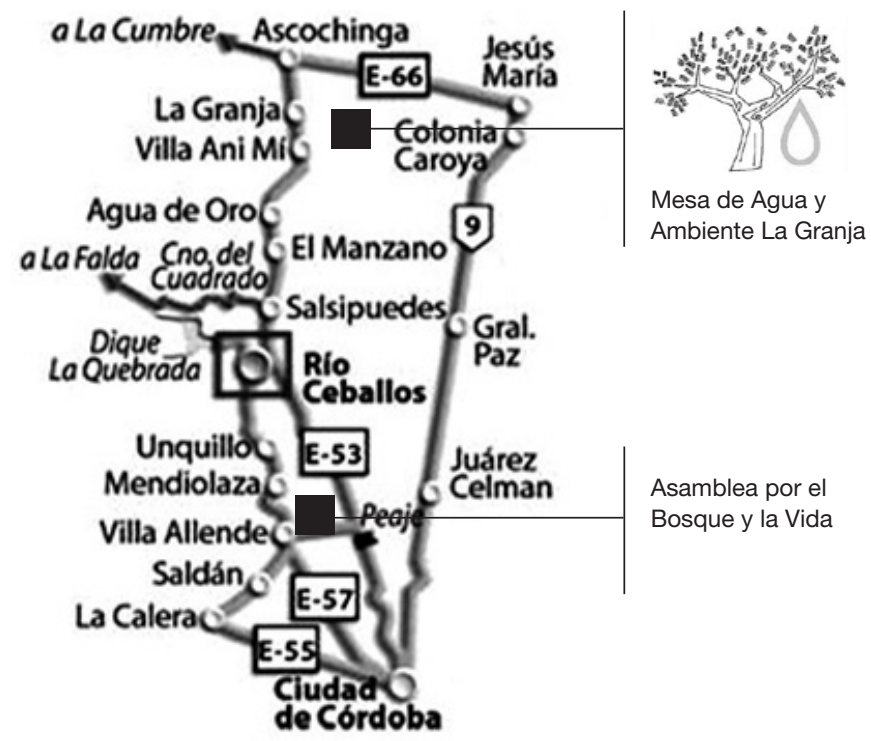

Las organizaciones sociales con las que se desarrollaron experiencias son La Mesa del Agua de La Granja, a 37 km de Córdoba capital, y la Asamblea de Villa Allende, a $25 \mathrm{~km}$. 
efectuamos las prácticas con referencia a su respectiva localidad. En las últimas décadas, en esta región se puso en evidencia una serie de problemáticas territoriales que, asociadas al cambio de uso del suelo, el alto crecimiento demográfico por migración y la presión inmobiliario-urbanística, llevaron a una fuerte degradación ambiental que tuvo su momento cúlmine el 15 de febrero de 2015, cuando todo el corredor se vio afectado por una inundación que dejó el saldo de 8 muertes y gravísimos daños materiales.

\section{Los problemas ambientales de la región y las organizaciones sociales}

Uno de los propósitos de la PSC, como se mencionó, es acercar la Universidad a los problemas concretos de la sociedad y poder colaborar en los procesos de reflexión crítica, identificación de problemas, diagnósticos o propuestas de resolución de conflictos, de manera conjunta con diferentes organizaciones sociales. Sobre la base de estos objetivos, ¿por qué seleccionamos y decidimos trabajar en la PSC con la Mesa del Agua y Ambiente de La Granja y con la Asamblea de Villa Allende? ¿Qué conocimiento teníamos como equipo de cátedra de las problemáticas locales/ regionales? ¿Qué acercamiento teníamos a las organizaciones sociales? ¿Cuánto conocíamos de su lucha?

Las experiencias de PSC que hemos venido desarrollando surgieron como corolario de un proceso conjunto de acciones entre el equipo de investigación "geografía del agua", del cual forma parte la mayoría de los miembros de la cátedra de Rural y los colectivos locales con los que hemos trabajado.

Ahora bien, ¿qué causas motivaron la organización de diferentes colectivos de Sierras Chicas? Aquí tenemos que ubicarnos en las condiciones ambientales de la región y principalmente el tema del agua. Las cuencas y microcuencas hídricas de la región sufren problemas como los recurrentes incendios, la creciente ocupación de espacios en urbanizaciones de distintos niveles (planes de vivienda, loteos abiertos, barrios cerrados, complejos turísticos), la deforestación y el sobrepastoreo. Estos factores, al limitar la capacidad de almacenamiento del agua y su liberación gradual, introducen cambios que implican la aceleración en el escurrimiento de las aguas superficiales de origen pluvial y producen inundaciones en épocas estivales lluviosas o una importante pérdida del recurso en temporadas secas.

Para comprender la complejidad de los procesos y las afectaciones a las poblaciones, desde un principio incorporamos al estudio de cuencas y microcuencas la dimensión del territorio, entendida como un entramado de relaciones sociales y de poder que se reproducen sobre el espacio al tiempo que lo producen. Los aspectos biofísicos, ecológicos y técnicos, no son suficientes para abordar un análisis que involucre las distintas dimensiones del territorio y es necesario incorporar las variables políticas, sociales y culturales, que son las que enmarcan los conflictos relacionados con este recurso al tiempo que lo convierten en lugar de construcción y refuerzo de asimetrías.

Lo anterior se encuadra en un entramado social complejo, que se ha ido imbricando con los cambios territoriales. Estas modificaciones traen aparejada otra concepción del recurso agua y de las prácticas relacionadas a su uso, a cargo de nuevos agentes que se asientan en la zona y que muchas veces entran en contradicción con las concepciones y prácticas de uso tradicional del agua, contradicciones que, en ocasiones, se expresan prácticas de agentes sociales con diferentes grados de poder y diversas intencionalidades. Así entendido, el problema hídrico de la vertiente oriental de las Sierras Chicas excede la superficie de las microcuencas y los límites jurisdiccionales de cada municipio y comuna. Se trata de un problema de carácter multidimensional y multiescalar que requiere de la consideración de sujetos sociales con diversos grados de agencia en el territorio donde se territorializan sus prácticas.

En este escenario, en la región de Sierras Chicas se han motorizado grupos de vecinos desde $2007 \mathrm{y}$, con diversos grados de organización, están llevando adelante importantes reclamos en defensa del agua, el suelo y el bosque. En definitiva, están poniendo en tensión las formas de apropiación, producción, distribución y gestión de los recursos naturales en cada localidad y a nivel regional. Luchan por mejores condiciones de vida, exigen mayor participación en las políticas públicas desde y en sus territorios. Este anclaje territorial tiene importancia para la ampliación de la democratización de nuestras sociedades. Concretamente, los múltiples colectivos de las diversas localidades regionales se han unido y organizado en la Coordinadora Ambiental y de Derechos Humanos de las Sierras Chicas ${ }^{4}$ y desde 2012 desarrollan acciones comunes tendientes a un trabajo en red:

"como deseo de implementar acciones conjuntas que permitan defender el ambiente de cada comunidad trabajando mancomunadamente las problemáticas ambientales de las Sierras Chicas en pos del desarrollo sustentable". (Eckstein, 2001:44)
4) Grupo EsCalera (La Calera), Fundación Vertientes de Saldán (Saldán), Grupo Tacku (Villa Allende), Guardianes del Monte (Mendiolaza), Vecinos Autoconvocados (Unquillo), Asociación
Civil Los Manantiales (Río Ceballos), CoVeSa (Salsipuedes), Fundación Eco-Barrio San Miguel (Salsipuedes), Coop. De Agua (Agua de Oro), Vecinos del Chavascate en Defensa del Agua, el Ambiente y la Calidad de Vida (Agua de Oro, Villa Cerro Azul), Colectivo Sin Frenos (Villa AniMi), Mesa del Agua de la Granja, Comunidad ContrArte, Grupo Coyuyo. 
Intentan otorgarle un sentido al ambiente, rescatar sus valores sociales como prestador de servicios ambientales; se trata de una lucha en común por los derechos sobre el agua, la tierra, el bosque y la identidad local.

\section{La búsqueda de soluciones multiescalares}

Como equipo de investigación y luego como cátedra incorporando a los alumnos, comenzamos a trabajar con las organizaciones realizando tareas como sistematización de datos, georreferenciación de áreas, entrevistas, trabajo de difusión en las localidades y en los medios académicos, definiciones de límites de cuencas bajas y altas de los ríos y sobre todo interpretación y aprehensión de conocimientos geográficos desde la experiencia y situación local, como forma de garantizar un discurso firme en la diálogo con otros agentes que influyen y deciden sobre el territorio. Avanzando en el tiempo, logramos elaborar propuestas y alternativas de solución más allá de las soluciones técnicas. Entre ellas se destacan: el impulso a la formación de un Corredor de Reservas Hídricas en las Sierras Chicas ${ }^{5}$ para proteger las cuencas de alimentación de los ríos serranos; la constatación de trasvase del río La Granja hacia valle de Punilla, puntualmente a la localidad de La Cumbre y posteriores negociaciones para recuperar la cuenca; y la declaración de Reserva Natural de la Defensa en los campos de la Fuerza Área en Ascochinga (diciembre de 2014).

En efecto, con el objetivo de mantener la superficie de absorción a través de la preservación del bosque nativo, en especial en las cuencas altas, y evitar mayores escorrentías a futuro, venimos trabajando junto a las organizaciones para la concreción de un corredor de reservas hídricas que contemple desde la Reserva Natural de la Defensa (RND) en los campos del III Cuerpo de Ejército en La Calera, ${ }^{6}$ al sur de la vertiente oriental de las Sierras, uniendo todas las áreas de reservas (la provincial de la Quebrada y las municipales) hasta el campo de aviación de Ascochinga, al norte. Esta última declarada en diciembre de $2014{ }^{7}$

El trabajo sobre las reservas hídricas, es una clara demostración de que es posible generar una serie de áreas naturales protegidas que, superando los límites político-administrativos de los municipios, permita crear un comité de cuencas de las Sierras Chicas que otorgue seguridad al bosque y proteja las cuencas altas de los ríos y arroyos ante el avance de la urbanización, los incendios, el desmonte para ganadería y nuevas urbanizaciones, así como los proyectos de trasvase de cuenca.

5) El Corredor Hídrico propuesto, se extiende desde Ascochinga hasta La Calera.

6) Se considera Reserva Natural de la Defensa (RND) a un tipo de Área Natural Protegida (ANP) creada por el gobierno nacional en el año 2007, tras firmarse el Convenio Marco de Coope-
El trabajo articulado de grupos de vecinos, ONG y comunas, permite que en 2014 se reglamenten la Reserva Los Manantiales (Río Ceballos) y Los Quebrachitos (Unquillo), se creen la Reserva para la Defensa de Ascochinga, la Reserva Hídrica y Natural de Villa Cerro Azul y se inicien acciones en la Reserva Hídrica y Recreativa Natural Villa Allende. Sobre las Reservas desarrollamos la PSC de 2016 y de 2017, que exponemos en el apartado siguiente.

\section{La propuesta didáctica de la PSC en el marco de la cátedra de Geografía Rural}

Como experiencia didáctica, y por tratarse de una propuesta curricular para estudiantes de grado, la PSC incluye instancias áulicas y extraáulicas, formas de evaluación y acreditación de los aprendizajes, todo ello durante un cuatrimestre.

Esquemáticamente, y haciendo el esfuerzo por sintetizar las experiencias desarrolladas en terreno, presentamos los pasos o momentos de la Práctica y las actividades realizadas.

\section{Primer momento: actividad aúlica}

Preparatoria del contacto en campo con la organización. Aquí se priorizan los contenidos que permitan a los estudiantes acercarse, comprender y sensibilizarse con aquellos problemas de la localidad, que constituyen el eje de lucha de la organización social con la que vamos a trabajar.

Los objetivos del Primer Trabajo Práctico son, en líneas generales:

- Comprender los problemas ambientales locales, sobre los que trabaja la organización social, como parte de situaciones conflictivas a escala regional.

- Reconocer, la historia del colectivo social, en su contexto de surgimiento, mantenimiento a través del tiempo, niveles de participación y modalidades de organización.

- Visualizar las acciones del colectivo social, como procesos de lucha que definen el grado de poder de los actores sociales sobre el territorio.

\section{Segundo momento: trabajo de campo en la localidad}

Se recorre el área, acompañados por gente de la organización, quienes guían el trayecto. Esta instancia es importante porque permite, en un ambiente informal, reconocerse mutuamente a los estudiantes y los miembros del colectivo.

Luego, en el salón de reuniones local, los integrantes de la organización relatan los hitos sobresalientes de la historia colectiva 
y seguidamente se comienza a elaborar un listado de temas prioritarios desde los cuales se negocia una agenda de trabajo con los estudiantes.

Los colectivos con los que articulamos plantean demandas y reclamos ambientales a través de lo que ellos denominan asociación/asamblea de autoconvocados y este formato posibilita una conversación horizontal abierta con los estudiantes desde cualquiera de los representantes del colectivo local.

Asignar un tiempo bastante prolongado para construir la demanda, los intereses, los problemas en conjunto con la organización, es relevante en la PSC ya que favorece la deconstrucción de algunos presupuestos relacionados con la Universidad como única portadora del saber, a la vez que se valoran los saberes locales. La actividad incluye, para los estudiantes, la entrega de un informe grupal sobre el registro del trabajo de campo, con eje en la conversación con el colectivo social.

\section{Tercer momento: actividad aúlica}

Preparatoria del taller a realizarse en la localidad. El Trabajo Práctico comienza con una puesta en común de los registros grupales de la primera experiencia en campo. A partir de estas reflexiones, se seleccionan los tópicos prioritarios entre la totalidad de demandas planteadas por la organización. Fundado en estas consideraciones se prepara el taller.

Nuestra experiencia nos muestra que los temas preocupantes, tanto para la MAyA como para la Asamblea de Villa Allende, son la participación y las tensiones entre discursos divergentes de los agentes sociales involucrados en la problemática. Asimismo, nos parece importante dotar de contenido los argumentos sostenidos en el espacio público por la organización, con relación a la defensa del ambiente. Esta mixtura de temas-problemas nos lleva a centrar nuestra atención y a profundizar en torno a los siguientes tópicos que constituyen la base del taller:

- Discursos y territorios

- Ambiente y cuestión ambiental

- Territorialidad y territorialización

- Agentes sociales y la conformación del territorio

- Bosque serrano y conservación

\section{Cuarto momento: taller en las localidades}

Los alumnos dirigen actividades grupales con participación de integrantes de las organizaciones colectivas locales. El taller es abierto a toda la comunidad, por ello suelen participar vecinos de la localidad y alumnos de las escuelas primaria y secundaria. El primer taller se denominó: "Participando, decidimos sobre nuestro territorio". Asistieron 120 personas que colmaron el comedor de la escuela primaria, Dr. Pablo Rueda, de la localidad de La Granja, el 22 de octubre de 2015. Los 35 estudiantes de la Facultad dividieron a los participantes en seis grupos donde se debatieron los temas: ambiente, territorio (cuencas hídricas), actores (roles y discurso), desarrollo.

Como conclusión del taller y articulando entre los grupos, se llegó a definir la complejidad de los distintos ambientes y su interacción con el hombre que tiene capacidad de agencia, es decir, que contribuye a la construcción de los espacios. También se comprendió el dinamismo de un territorio, sobre todo del territorio-cuenca y la importancia de definir sus límites y la necesidad de ordenarlo con base en el recurso agua y su justa distribución.

El segundo taller se llevó a cabo con la MAyA, representante de Parques Nacionales y de la Fuerza Área, organismo que administra la Reserva Natural de Ascochinga. El tema fue: "Impactos socioambientales y resistencias locales por el proyecto de construcción de minidique en la Reserva Natural Ascochinga", realizado el 2 de junio de 2016.

Como producto del taller se realizó una Declaración conjunta entre la MAyA y cátedra de Rural de la UNC, en contra del proyecto del gobierno provincial, de construcción de un dique sobre el curso del río Carapé.

Además, se elaboró un documento colectivo (profesores, miembros de la MAyA, de la Reserva Natural y de Parques Nacionales) para participar en la audiencia pública que se llevó a cabo en la Sociedad Rural de Jesús María, cabecera del departamento Colón, el 13 de junio de 2016. Hasta el momento, no se ha realizado ninguna obra sobre los arroyos de la Reserva Natural Ascochinga. Además, lo que fue anunciado públicamente como una obra inminente, con fondos de la Nación, con estudios de impacto ambiental presentados y aprobados por la Secretaría de Ambiente de la provincia, se silenció absolutamente y no se mencionó más el tema desde los órganos oficiales. La MAyA continúa su lucha y recuerda los movidos días de junio como testimonio de que la organización y participación colectiva en defensa del ambiente a veces tiene sus frutos.

El tercer taller se realizó el 1 de junio de 2017 en la escuela General José de San Martín, titulado: "El territorio en disputa. Problemáticas relacionadas al uso del suelo en Villa Allende". Se produjo cartografía específica de tipo participativa del área en conflicto, es decir, las 400 has amenazadas con emprendimiento inmobiliario dentro de la Reserva Municipal "San Felipe".

Se trabajó con los múltiples factores que han producido el crecimiento urbano de Villa Allende y han desembocado en serios problemas de injusticia ambiental.

Se planteó la necesidad de realizar un proyecto de Ordenamiento Territorial Participativo, en donde cada vecino, cada empleado público e incluso aquellos actores privados que tengan interés en la zona, participe, discuta y ponga en tensión sus argumentos. No olvidemos que el territorio es una construcción social por parte de agentes con distintos grados de acción y decisión. En este proceso, la Universidad Nacional también debería asistir para aportar sus conocimientos teórico-prácticos y para que, en forma 
interdisciplinaria y sumados estos al saber que poseen aquellos que viven el lugar, se pueda llegar por fin a diseñar alternativas más amigables con el ambiente y que permitan una mejor calidad de vida a los habitantes.

El conocimiento y divulgación del conflicto debe llegar a cada escuela, cada hogar, cada institución, porque el espacio es de todos, la tierra es un bien social, por eso, antes de utilizarla, debemos considerar los efectos de nuestra actividad.

Una de las conclusiones fue que no se conoce bien el lugar donde se quiere extender la urbanización. Para ello se propuso el armado de actividades desde los colegios, como puede ser reforestar la zona, realizar actividades recreativas dentro de la Reserva y también caminatas los sábados por el lugar, organizadas por el grupo Tacku.

\section{Quinto momento: actividad de cierre de las PSC}

Habitualmente se realiza una relatoría de la experiencia y elaboración de informe final en una mesa de diálogo a cargo de la cátedra, los estudiantes, la Secretaría de Extensión de la Facultad y miembros de las organizaciones con las que se trabajaron.

Esta actividad se hace en contracuatrimestre y tiene carácter de evaluación final, con acreditación.

Algunas reflexiones surgidas de esta actividad sirven como un balance de las experiencias. Con relación a la implementación, queremos marcar como necesario asignar un tiempo bastante prolongado para construir la agenda de trabajo en conjunto con la organización.

Por su parte, los estudiantes destacan la dificultad presentada a la hora de sostener el ritmo de lectura propuesto por la cátedra a la par del trabajo con la institución u organización, aunque remarcaron la relevancia de la instancia como experiencia formativa. En este sentido, proponen la creación de espacios curriculares consecutivos y correlativos en vínculo con la misma institución u organización, en donde pudieran insertarse para continuar con la experiencia al menos por un período anual. Asimismo, remarcan la importancia de que la totalidad de los actores locales formen parte activa del proceso que involucra la Práctica para asegurar el interés y la participación sostenida por parte de estos.

Los miembros del colectivo social plantean la importancia de mantener una actitud abierta de su parte, así como de los universitarios, a la hora de reconocer la historia previa al trabajo común y los conocimientos construidos en ese proceso. La importancia de los aprendizajes mutuos queda en parte reflejada en el testimonio de una practicante:
"Estar en el lugar, poder debatir la problemática con la gente, es una fuente de información, pero además es indispensable para el enriquecimiento del trabajo. Sin esta experiencia, no se llegarían a abarcar las voces de todos los involucrados, dejando actores claves invisibilizados para aportar solución a los problemas. El debate con la gente de la MAyA enriqueció nuestros conocimientos, por eso afirmo que es el punto de partida fundamental para el intercambio de saberes y experiencias!". (Fernanda Sosa, 2015).

También se visualiza la importancia de la PSC para la gente de la organización en este fragmento que hemos seleccionado:

"En el taller con la gente de la universidad, aportamos y compartimos experiencias, inquietudes y conocimientos. Estamos agradecidos por el compromiso y dedicación de los profesores y estudiantes de geografía, queremos mantener este vínculo ya que, para nosotros, es difícil acercarnos y trabajar con los universitarios, y lo hemos logrado a través de la PSC". (Christine, La Granja, 2016).

En definitiva, creemos que a través de la PSC que es posible ampliar los esquemas interpretativos de la realidad en disputa, posibilidad que se abre a todos los que participan: docentes, estudiantes, organizaciones.

La PSC ha tenido impacto entre los estudiantes de Geografía, más allá de la cátedra de Rural. Nos estamos refiriendo a la opción de estas temáticas para Trabajo Final de Grado (TFG) o Práctica Preprofesional Supervisada (PPS). Así es que contamos hasta el presente con dos Trabajos Finales aprobados:

- Gestión de cuenca. Conflictos por el uso del suelo y el agua en las Sierras Chicas: el caso de la cuenca del río Chavascate, Agua de Oro, Córdoba (Argentina).

- Espacialidades, políticas y sentido de lugar en las reivindicaciones de los actores organizados en torno a la Reserva Bamba, La Calera (Córdoba) 2005-2016

Y los siguientes proyectos:

- Conflictos por los territorios: usos del agua y del suelo en Sierras Chicas (PPS).

- Relevamiento y mapeo integral de la Reserva Natural Urbana Río La Granja. Utilizando Sistemas de Información Geográfica y herramientas de Teledetección (PPS).

- Diagnóstico de la situación ambiental en Ascochinga, con especial referencia a la Reserva Natural de la Defensa. Evolución, conservación y las problemáticas actuales (TFG). 

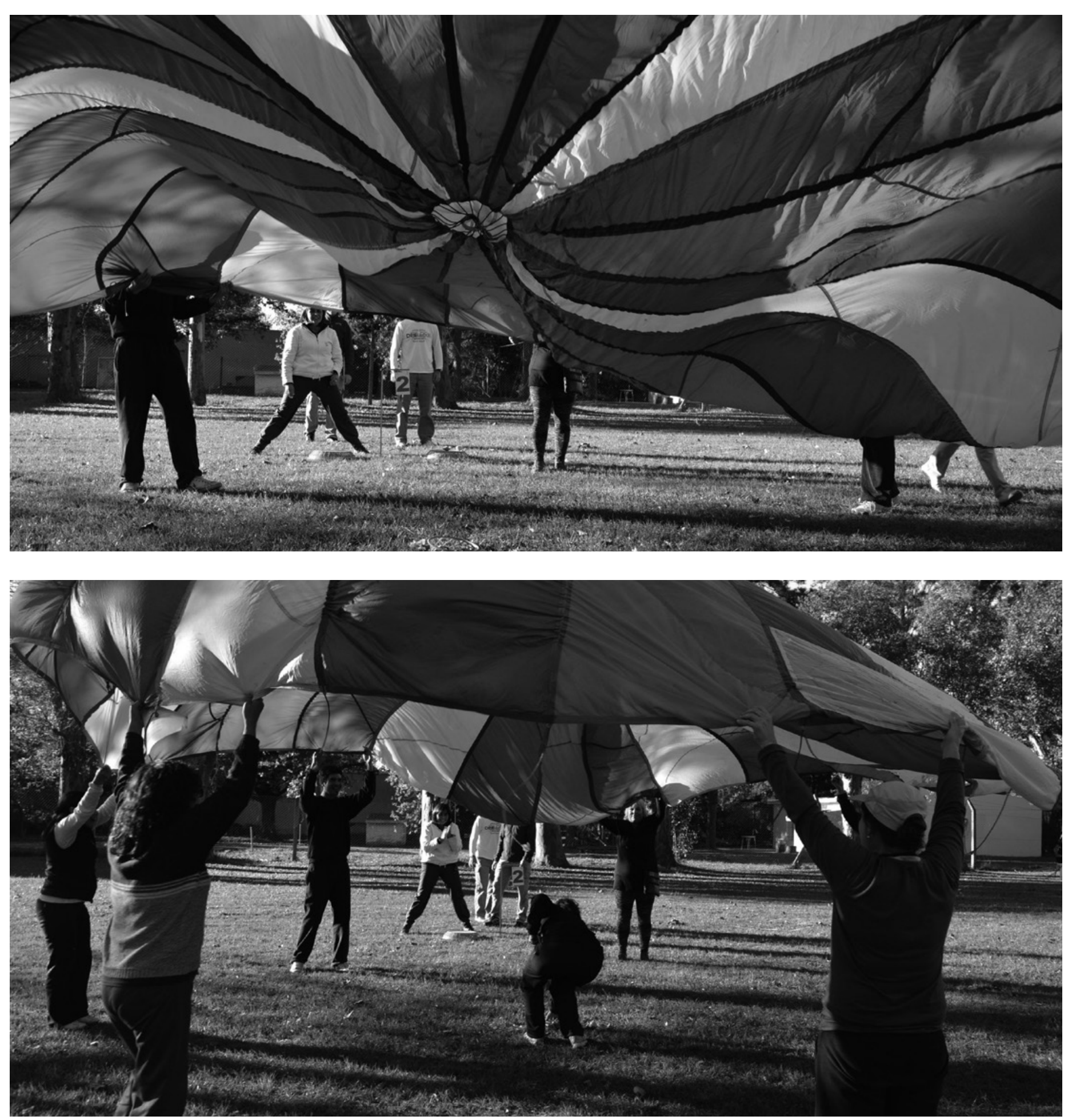

(C) Laura Malachesky 


\section{A manera de cierre, algunas reflexiones}

El anclaje socioterritorial de las organizaciones sociales y el trabajo participativo desde la Universidad a través de PSC tienen importancia decisiva para la democratización de la sociedad, además de su valor como innovación pedagógica en el ámbito de la FFyH. El trabajo de campo lo desarrollamos en un área conflictiva del Gran Córdoba, como es la de las Sierras Chicas. Los problemas de sequías e inundaciones dan cuenta claramente de una zona problemática que necesita la búsqueda de soluciones multiescalares y la participación de actores provenientes de distintos ámbitos, interesados en desplegar acciones compartidas en el territorio concreto del conflicto.

En este sentido, en torno al ambiente, se están construyendo nuevos espacios públicos en el nivel microlocal a partir de las prácticas de las organizaciones comunitarias.

La unión en los últimos años de las agrupaciones y organizaciones sociales a nivel local ha activado mecanismos de visibilización de los conflictos. Sus acciones van más allá de los límites políticos de cada jurisdicción y, como toda construcción política, la lucha comunitaria muestra logros, dificultades y contradicciones. La perspectiva desde la cual sostenemos la PSC nos permite vivenciar la co- construcción del conocimiento social. A través del intercambio producido con la PSC se conoce el territorio, se experimenta el trabajo de campo, se aprende a construir conocimiento desde la fusión entre lo experiencial y lo académicoformal. A su vez, enriquecemos los marcos teóricos de los sujetos, tanto los locales como los universitarios, al intercambiar conceptos ordenadores sobre los problemas y conflictos de la realidad. Entendemos el aprendizaje de los futuros geógrafos como una forma de trabajo colaborativo entre practicantes, abonando espacios de diálogo entre los actores sociales.

Trabajar con organizaciones sociales de base territorial motorizadas, que conocen los problemas de su localidad en profundidad, permite a los alumnos, entre otros aprendizajes valiosos, acercarse a la realidad del conflicto desde la visión de sus protagonistas y comprender que el campo social donde se construye la lucha por la defensa del ambiente es un camino con marchas y contramarchas, donde la participación y el compromiso de los que luchan tienen sus vaivenes, sus encuentros y desencuentros. Permite observar que las posiciones de los actores en el conflicto se van construyendo en el proceso mismo de despliegue de la acción colectiva y no previamente a partir de una posición unificada; esta dinámica es la que va generando formas organizacionales y lazos institucionales.
Pudimos y podemos valernos del formato asambleario de las organizaciones para impulsar deliberaciones grupales sobre diferentes tópicos, que amplíen la exploración de argumentos tanto para alumnos como para la organización y el pueblo en general, tomando en cuenta una pluralidad de puntos de vista, demandas y posibles respuestas, todo ello considerado como aprendizaje social. En la práctica, al poner en juego los diversos discursos que sostienen los agentes en el territorio, se puede discutir y sacar nuevas conclusiones.

A través del acercamiento a actores e instituciones del orden local, se comparten problemáticas, experiencias y saberes en un proceso de construcción conjunta, tendiente a la constitución de un espacio de intercambio de conocimientos entre actores sociales que vienen trabajando en la región. Por eso planteamos la PSC como una manera de comenzar a impulsar lo que Callon et al. denominan "dispositivos de exploración y de aprendizaje colectivo". La necesidad de poner en juego los conocimientos validados por la academia es lo que lleva al intercambio y encuentro con la gente organizada de La Granja y de Villa Allende. Siguiendo nuevamente a Callon et al: :

"en aquellas situaciones donde hay un foco de disputa por un daño o afectación ambiental, se pone en discusión qué tipo de conocimiento será admitido como prueba y quiénes pueden ser los actores acreditados para generar ese conocimiento". (2001:47-50)

Por último, queremos apuntar que nuestra PSC no sería posible sin el apoyo de la Secretaría de Extensión de la Facultad, responsable de la creación, impulso y sostenimiento de las Prácticas como modalidad de formación de grado. Creemos que con este proyecto en acción, desde esta Secretaría, dan respuesta a lo que Boaventura de Sousa Santos imagina para la universidad del futuro, donde se otorgue un lugar central a las tareas de extensión como una manera de achicar la brecha entre la ciencia, el conocimiento académico y los problemas y necesidades reales de la sociedad.

"En el momento en que el capitalismo global pretende reducir la universidad en su carácter funcionalista y transformarla de hecho en una amplia agencia de extensión a su servicio, la reforma de la universidad debe conferir una nueva centralidad a las actividades de extensión, con implicaciones en el currículo y en las carreras de los docentes". (De Sousa Santos, 2005:31). 


\section{Referencias bibliográficas}

Callon, M.; Lascoumes, P. y Barthe, Y. (2001). Agir dans un monde incertain. Essai sur la démocratie technique. Paris: Seuil.

Chiavassa, S. (2009). La extensión, un lugar en geografía. Revista e+e. Estudios de extensión en humanidades, 88-97. FFyH/UNC.

Eckstein, S. (Coord.) (2001). Poder y protesta popular en América Latina. México: Siglo XXI Editores.

Escobar, A. (1996). La invención del Tercer Mundo. Construcción y deconstrucción del desarrollo. Bogotá: Norma.

De Sousa Santos, B. (2003). Crítica de la razón indolente. Volumen 1. Para un nuevo sentido común: La ciencia, el derecho y la política en la transición paradigmática. Bilbao: Desclee.

(2005). La universidad en el siglo XXI. Para una reforma democrática y emancipadora de la universidad. Umbrales, (15, marzo), 1-58. CIDES, Postgrado en Ciencias del Desarrollo, UMSA, Universidad Mayor de San Andrés, La Paz, Bolivia. Disponible en: http://biblioteca.clacso.edu.ar/ar/libros/bolivia/cides/umbrales/15/umbrales15.html

Merlinsky, G. (Coord.) (2013). Cartografía de los conflictos ambientales en Argentina. Buenos Aires: CLACSO.
Vasilachis, I. (1999). Trabajo y Derecho. En Agulla, J. C. (Comp.), Ciencias Sociales: presencia y continuidades. Buenos Aires: Academia Nacional de Ciencias de Buenos Aires. Instituto de Derecho Público, Ciencia Política y Sociología.

Zaffaroni, A. y Juarez, C. (2014). Políticas de lugar, ecología de saberes y pensamiento epistémico en experiencias de coinvestigación en el NOA. Cardinalis, 2(2, primer semestre), 99-116. Córdoba: UNC.

Zemelman, H. (2011). La postura epistemológica de Hugo Zemelman. Conocimiento y Ciencias Sociales. Contribución al estudio del presente. La Paz: Convenio Bello.

\section{Documentos}

Proyectos de PSC de la cátedra de Geografía Rural del Departamento de Geografía (2014-2016-2017) aprobados por la Secretaría de Extensión y la Secretaría Académica de la FFyH. Formularios pdf.

Reglamento Práctica Sociocomunitaria, FFyH, UNC. Resolución del Honorable Consejo Directivo N²26/2013. Disponible en: http://www.ffyh.unc.edu.ar/extension/wp-content/uploads/2013/10/RES_226_2013.pdf 